\title{
Ultrashort dead time of photon-counting InGaAs avalanche photodiodes
}

A. R. Dixon, J. F. Dynes, Z. L. Yuan (袁之良), A. W. Sharpe, A. J. Bennett, and A. J. Shields

Citation: Appl. Phys. Lett. 94, 231113 (2009);

View online: https://doi.org/10.1063/1.3151864

View Table of Contents: http://aip.scitation.org/toc/apl/94/23

Published by the American Institute of Physics

\section{Articles you may be interested in}

High speed single photon detection in the near infrared

Applied Physics Letters 91, 041114 (2007); 10.1063/1.2760135

Gigahertz-gated InGaAs/InP single-photon detector with detection efficiency exceeding $55 \%$ at $1550 \mathrm{~nm}$ Journal of Applied Physics 117, 083109 (2015); 10.1063/1.4913527

Single-photon detection efficiency up to $50 \%$ at $1310 \mathrm{~nm}$ with an InGaAs/InP avalanche diode gated at 1.25 $\mathrm{GHz}$

Applied Physics Letters 102, 141104 (2013); 10.1063/1.4801939

Invited Review Article: Single-photon sources and detectors

Review of Scientific Instruments 82, 071101 (2011); 10.1063/1.3610677

Multi-gigahertz operation of photon counting InGaAs avalanche photodiodes

Applied Physics Letters 96, 071101 (2010); 10.1063/1.3309698

Fully integrated InGaAs/InP single-photon detector module with gigahertz sine wave gating

Review of Scientific Instruments 83, 083111 (2012); 10.1063/1.4746291

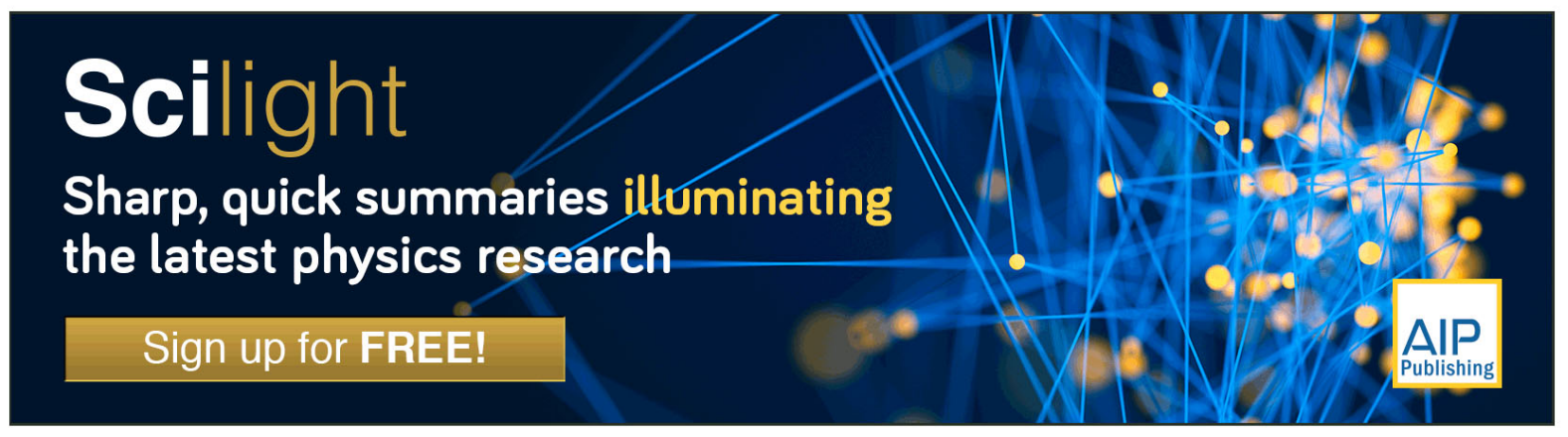




\title{
Ultrashort dead time of photon-counting InGaAs avalanche photodiodes
}

\author{
A. R. Dixon, ${ }^{\text {a) }}$ J. F. Dynes, Z. L. Yuan (袁之良), ${ }^{\text {b) }}$ A. W. Sharpe, A. J. Bennett, and \\ A. J. Shields \\ Toshiba Research Europe Ltd., Cambridge Research Laboratory, 208 Cambridge Science Park, Milton \\ Road, Cambridge CB4 OGZ, United Kingdom
}

(Received 3 March 2009; accepted 15 May 2009; published online 9 June 2009)

\begin{abstract}
We report a $1.036 \mathrm{GHz}$ gated Geiger mode InGaAs avalanche photodiode with a detection dead time of just $1.93 \mathrm{~ns}$. This is demonstrated by full recovery of the detection efficiency two gate cycles after a detection event, as well as a measured maximum detection rate of $497 \mathrm{MHz}$. As an application, we measure the second order correlation function $g^{(2)}$ of the emission from a diode laser with a single detector that works reliably at high speed owing to the extremely short dead time of the detector. The device is ideal for high bit rate fiber wavelength quantum key distribution and photonic quantum computing. (C) 2009 American Institute of Physics. [DOI: 10.1063/1.3151864]
\end{abstract}

Many important applications, such as biological imaging, ${ }^{1}$ fiber optical sensing, ${ }^{2}$ laser ranging, ${ }^{3}$ and quantum communication, ${ }^{4}$ require high performance single photon detectors, for which semiconductor avalanche photodiodes ${ }^{5-8}$ (APDs) have proved to be a practical choice due to their compactness, cryogenic-free operation, and low power consumption. Unfortunately, APDs are often associated with a long dead time, which is particularly true for InGaAs APDs. This directly limits performance of a photon counting based system. More seriously, for applications such as quantum key distribution, excessively long dead time can be exploited by an adversary, potentially compromising of the system security. ${ }^{9,10}$ Long dead time can be mitigated through the use of a detector array switched actively ${ }^{11}$ or passively. ${ }^{12}$ However, these solutions either add to the system complexity ${ }^{11}$ or increase the dark count rate. ${ }^{12}$

We recently demonstrated a promising technology for high speed single photon detection with InGaAs/InP APDs. ${ }^{8}$ A self-differencing (SD) circuit that compares the APD output with that in the preceding period allows detection of extremely weak avalanches, thus enabling fast, efficient, low-noise single photon detection. A $100 \mathrm{MHz}$ photon count rate has been achieved but its full potential was prevented by limitations in the photon counting electronics. Although SDAPDs have already been successfully applied to high bit rate quantum key distribution ${ }^{13,14}$ and quantum random number generation, ${ }^{15}$ it remains desirable to experimentally determine their ability to perform photon counting.

In this letter, we study experimentally the photon counting dead time using a high bandwidth oscilloscope, which eliminates the speed deficiencies associated with photon counting electronics. The dead time for an SD InGaAs APD operating at a gating frequency of $1.036 \mathrm{GHz}$ is determined to be 1.93 ns. Thanks to the short dead time, we demonstrate a count rate of $497 \mathrm{MHz}$ at a high detection efficiency of $14 \%$. Such an ultrashort dead time makes it possible to use a single detector in applications that generally require two or more. A single detector Hanbury-Brown and Twiss (HBT) experiment ${ }^{16}$ is realized to measure the second order corre-

\footnotetext{
a) Also at Cavendish Laboratory, University of Cambridge, J. J. Thomson Avenue, Cambridge CB3 OHE, UK.

b) Author to whom correspondence should be addressed. Electronic mail: zhiliang.yuan@crl.toshiba.co.uk.
}

lation of the emission from an attenuated diode laser.

As an important parameter, dead time deserves a clear definition. It is usually regarded as the time after a photon detection event during which the detector is unable to register another photon. Here, we define dead time as the smallest time duration after which the detection efficiency is independent of previous photon detection history. In other words, a detector should recover to its intrinsic detection efficiency within the dead time after a previous photon detection. In principle, an SD-APD is able to produce an avalanche within every gating period. However, due to the self-differential nature, an avalanche signal will be canceled out if there is an avalanche in its preceding gate. This renders a theoretical dead time of two gating periods for an SD detector.

To determine the dead time, we illuminate the device with a train of double pulses ${ }^{17}$ with variable time separation $(\Delta t)$, see Fig. 1(a) inset. The single photon detector under study is an InGaAs APD cooled electrically to $-30{ }^{\circ} \mathrm{C}$. It is operated in SD mode with a $1.036 \mathrm{GHz}$ voltage square wave gating. A 15 ps pulsed laser at a wavelength of $1550 \mathrm{~nm}$ is used for illumination. Two pulses of equal intensity and variable time separation, with a repetition rate of $16.19 \mathrm{MHz}$ (1.036 GHz/64), are achieved through use of external intensity modulation of the pulsed laser. Since only photons arriving within a detector gate can be detected, ${ }^{8}$ the laser and the detector gating are synchronized with their relative delay tuned to ensure efficient photon detection. The detection efficiency is set to be about $10 \%$ by tuning the APD DC bias. Under such conditions, the afterpulse probability is about $5 \%$ integrated over many gates thus becomes negligibly small for each individual gate.

We first examine the SD-APD dead time. An oscilloscope is used to record single shot waveforms of the APD output. A typical waveform displaying detection of both optical pulses is shown in Fig. 1(a). Repeated measurements allow us to determine probabilities for detecting each pulse individually or both together. Detection probabilities are shown in Fig. 1(b) as a function of the incident photon flux for $\Delta t=1.93 \mathrm{~ns}$. Also plotted is a theoretical calculation assuming that detections for pulses 1 and 2 are independent of each other. Here we list experimental observations:

(1) $P_{1}=P_{2}$ within experimental uncertainty, where $P_{1}$ and $P_{2}$ are detection probabilities for detecting pulses 1 and 

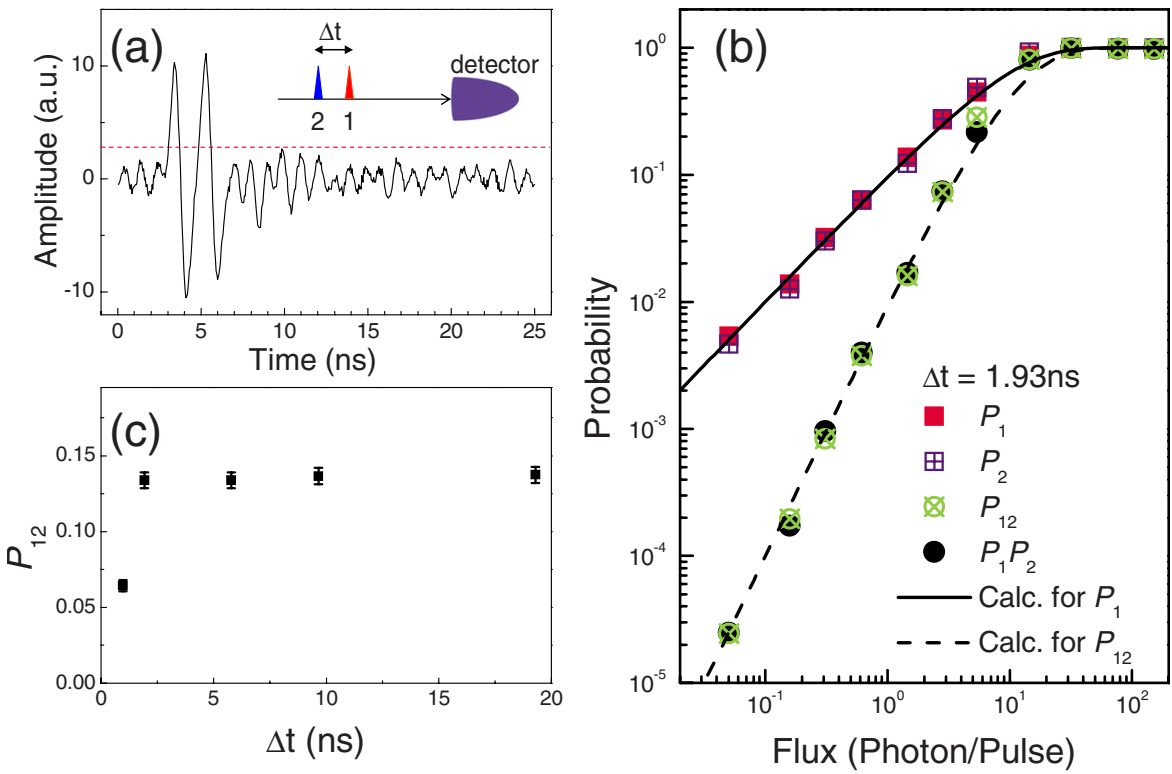

FIG. 1. (Color online) Experimental verification of the ultrashort detector dead time at a detector gating clock of $1.036 \mathrm{GHz}$ using a double pulse method as schematically shown in the inset of (a). (a) Single shot output of an SD-APD recorded by an oscilloscope, displaying detection of both optical pulses separated by 1.93 ns. (b) Probabilities for detecting the first pulse $\left(P_{1}\right)$, the second $\left(P_{2}\right)$, and both simultaneously $\left(P_{12}\right)$. Also shown is product of $P_{1}$ and $P_{2}$. The lines show the theoretical calculation using a detection efficiency of $10 \%$, assuming no influence from detector dead time. (c) Joint detection probability as a function of the time delay between the two pulses. The illumination intensity corresponds to a single pulse detection probability of 0.36 .
2 respectively; The result $P_{1}=P_{2}$ suggests that detector afterpulsing is negligible.

(2) $P_{1}$ and $P_{2}$ are linearly dependent on the incident photon flux before detector saturation.

(3) $P_{12}=P_{1} P_{2}$, where $P_{12}$ is the probability for simultaneous detection of both pulses.

(4) All the measured parameters agree well with calculation using a photon detection efficiency of $10 \%$.

Based on the above observations, we can deduce the conditional detection probability $P_{2 \mid 1}$, i.e., the detection probability of pulse 2 given pulse 1 is detected, as

$$
P_{2 \mid 1}=P_{12} / P_{1}=P_{1} P_{2} / P_{1}=P_{2} .
$$

The result $P_{211}=P_{2}$ states that detection of pulse 1 does not affect detection probability of pulse $2.1 .93 \mathrm{~ns}$ after detection of pulse 1 , the detector has already recovered to its detection efficiency of $10 \%$. This exactly satisfies our definition for the dead time. We can therefore conclude that the SD-APD operating at $1.036 \mathrm{GHz}$ has a dead time of $1.93 \mathrm{~ns}$.

Figure 1(c) shows the joint detection probability $P_{12}$ as a function of the time delay $\Delta t$ measured at a fixed photon flux. The joint probability does not depend on the time delay provided that $\Delta t$ is not smaller than two gating periods, illustrating again that the experimentally determined $1.93 \mathrm{~ns}$ is indeed the detector dead time. $P_{12}$ is significantly smaller when $\Delta t$ is one gating period, which is smaller than the detector dead time. This is due to cancellation of avalanches in consecutive gating periods by the SD circuit. $P_{12}$ does not fall to zero because of incomplete cancellation of the avalanche signals, for which the amplitude distribution is photon number dependent. ${ }^{18}$

A short dead time is a necessary but not sufficient condition to achieve a high count rate. To examine the detection rate limit, the SD-APD is illuminated by an attenuated 1550 $\mathrm{nm}$ pulsed laser with a repetition rate of $518 \mathrm{MHz}$, corresponding to an illumination pulse every other detector gate. The measured count rate is plotted in Fig. 2 as a function of the incident photon flux, together with a theoretical calculation using a detection efficiency of $14 \%$ and a dark count probability of $1.67 \times 10^{-5}$ per gate. The observed count rate agrees well with the theoretical curve, both displaying a lin- ear dependence on the incident flux before saturation. Under strong illumination ( $>10$ photons/pulse), the measured count rate saturates at $497 \mathrm{MHz}$, reaching $96 \%$ of its dead time limited capacity of $518 \mathrm{MHz}$ being half the detector clock frequency of $1.036 \mathrm{GHz}$. The small discrepancy (4\%) is attributed mainly to afterpulses ${ }^{8}$ in nonilluminated gates, which cancel a fraction of photon-induced avalanches.

A few remarks are needed for the achieved dead time and count rate. (1) The demonstrated dead time is the shortest achieved so far for any semiconductor based detector system. It is more than one order of magnitude shorter than APDs operating in an active quenching mode..$^{5}$ (2) Not only is the demonstrated $497 \mathrm{MHz}$ the highest count rate achieved so far for any single element APD but more importantly also exceeds the value obtained by an APD array of 132 elements. ${ }^{12}$ (3) The APD outperforms photomultiplier tubes in photon count rate. ${ }^{19}$ It also compares favorably with cryogenic superconducting nanowire based detectors, whose inductance-limited recovery time usually supports a photon count rate not greater than $120 \mathrm{MHz}$ at an optimized efficiency. ${ }^{17}$ (4) The count rate can be further increased by

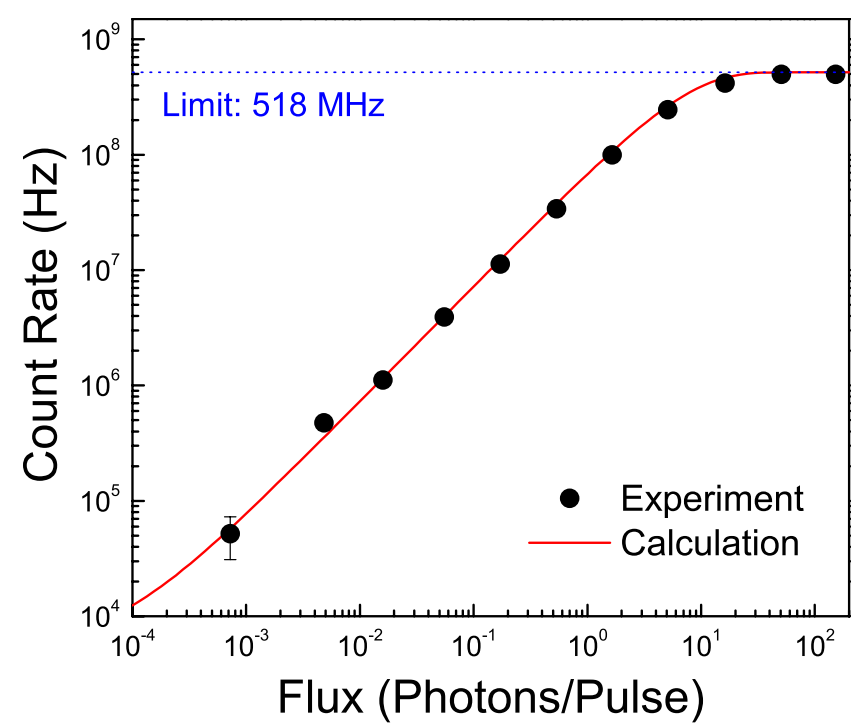

FIG. 2. (Color online) Experimental and calculated count rates vs incident photon flux. The dashed line shows the dead time limited count rate. 


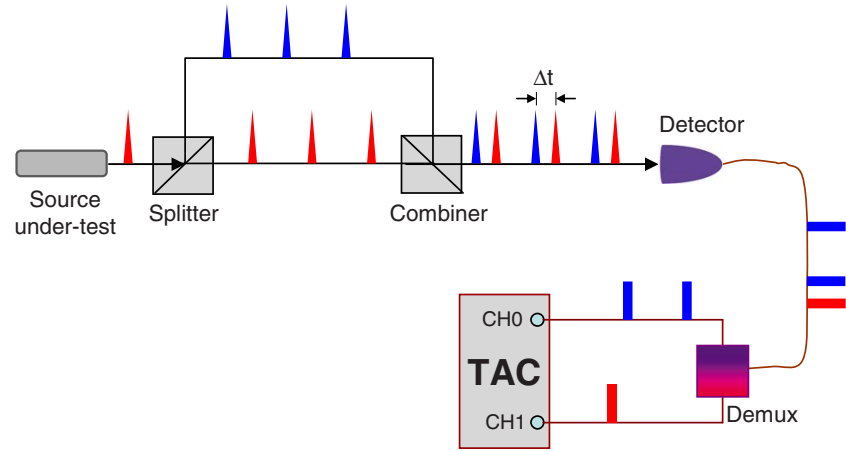

FIG. 3. (Color online) Configuration for the second order correlation $g^{(2)}$ measurement of a pulsed source with a single detector. Before the time acquisition card (TAC), a demultiplexer (Demux) is used to separate electrical signals due to photons that passed through different paths of the asymmetric Mach-Zender interferometer.

operating the APD at an even higher clock frequency. As a compact semiconductor device, APDs will play a significant role in applications where high count rate is of paramount importance.

Apart from offering high bit rates, ${ }^{13-15}$ a high speed device is also useful for applications that conventionally require two or more detectors. As an illustration, a single detector is sufficient to measure the second order correlation $g^{(2)}(t)$ of a pulsed source using a modified HBT setup. The trick here is to time-multiplex optical signals so that two beams can effectively be detected by a single detector. As shown in Fig. 3, a pulsed source under test is split and recombined using an asymmetric Mach-Zender interferometer. The time difference $\Delta t$ between two optical arms corresponds to an integer number of detector gating periods. The recombined beam is detected by an SD-APD, whose electrical output is demultiplexed before analysis by time correlation electronics.

A semiconductor diode laser heavily attenuated to single photon level is tested in the single detector HBT setup with $\Delta t=4 \mathrm{~ns}$. Driven at $125 \mathrm{MHz}$ by a voltage pulse generator, the laser emits a number of axial modes across a spectral region of 1540-1565 $\mathrm{nm}$. Its power dependence on driving voltage indicates a lasing threshold of $1.20 \mathrm{~V}$, as shown in Fig. 4(a). When biasing the diode well above its threshold, the measured second order correlation function gives $g^{(2)}(0)=1.0$, as shown in Fig. 4(b). The unity $g^{(2)}$ is consistent with a laser source that obeys Poissonian photon number statistics for above threshold operation.

When operating the diode at its lasing threshold, the laser intensity is expected to fluctuate. Such fluctuation can be revealed experimentally as bunching $\left[g^{(2)}(0)>1\right] .{ }^{20}$ The bunching effect is more significant in the output of each individual axial mode because competition for intensity among the modes further increases fluctuation. We therefore measure $g^{(2)}$ for a spectrally filtered mode. As expected, the measured $g^{(2)}$ displays a strong bunching with $g^{(2)}(0)=1.4$, as shown in Fig. 4(c). Note that the $g^{(2)}$ measurement results are in excellent agreement with those obtained with a conventional HBT setup consisting of two detectors (data not shown here) thus corroborating that the single detector HBT setup has faithfully measured the second order correlations.

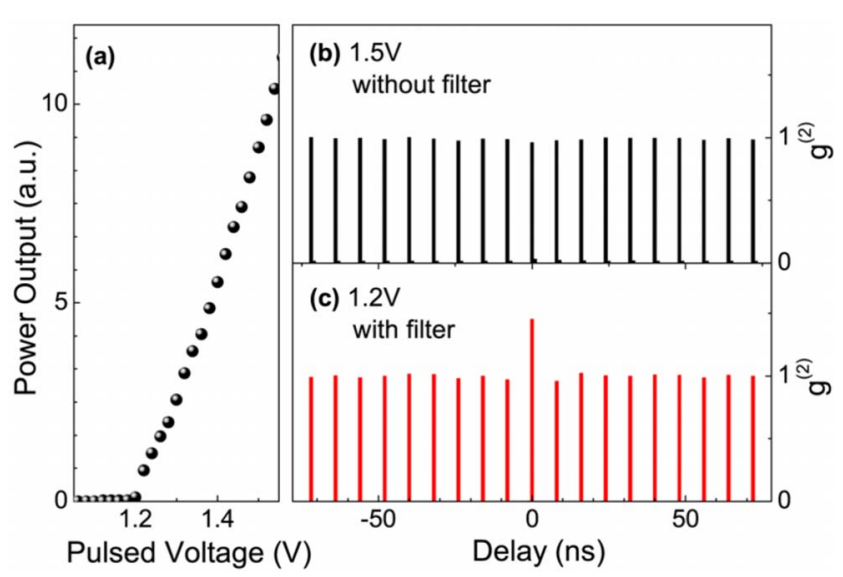

FIG. 4. (Color online) Second order correlation function $g^{(2)}(t)$ measurements by a single detector HBT configuration shown in Fig. 3 for a semiconductor diode laser pulsed at $125 \mathrm{MHz}$. (a) Laser output power as a function of driving pulse voltage. (b) $g^{(2)}$ measured for the diode biased at $1.5 \mathrm{~V}$. (c) $g^{(2)}$ measured for a spectrally filtered mode of the diode biased at the lasing threshold.

In summary, we have determined experimentally an ultrashort dead time of less than $2 \mathrm{~ns}$ and a photon count rate of nearly $500 \mathrm{MHz}$ for an InGaAs APD. The short dead time is important for high bit rate single photon applications, such as quantum key distribution and photonic quantum computing. The detector may also be used for recording secondorder correlations using a single detector.

${ }^{1}$ K. Suhling, J. Siegel, D. Phillips, P. M. W. French, S. Leveque-Fort, S. E. D. Webb, and D. M. Davies, Biophys. J. 83, 3589 (2002).

${ }^{2}$ J. M. Lopez-Higuera, Handbook of Optical Fiber Sensing Technology (Wiley, Chichester, 2002).

${ }^{3}$ P. A. Hiskett, C. S. Parry, A. McCarthy, and G. S. Buller, Opt. Express 16, 13685 (2008).

${ }^{4}$ C. Gobby, Z. L. Yuan, and A. J. Shields, Appl. Phys. Lett. 84, 3762 (2004)

${ }^{5}$ S. Cova, M. Ghioni, A. Lotito, I. Rech, and F. Zappa, J. Mod. Opt. 58, 1267 (2004)

${ }^{6}$ A. Yoshizawa, R. Kaji, and H. Tsuchida, Jpn. J. Appl. Phys., Part 2 43, L735 (2004).

${ }^{7}$ N. Namekata, S. Sasamori, and S. Inoue, Opt. Express 14, 10043 (2006).

${ }^{8}$ Z. L. Yuan, B. E. Kardynal, A. W. Sharpe, and A. J. Shields, Appl. Phys. Lett. 91, 041114 (2007).

${ }^{9}$ V. Makarov, arXiv:0707.3987v3.

${ }^{10}$ D. J. Rogers, J. C. Bienfang, A. Nakassis, H. Xu, and C. W. Clark, New J. Phys. 9, 319 (2007).

${ }^{11}$ S. Castelletto, I. P. Degiovanni, V. Schettini, and A. Migdall, J. Mod. Opt. $\mathbf{5 4 ,} 337$ (2007).

${ }^{12}$ P. Eraerds, M. Legré, A. Rochas, H. Zbinden, and N. Gisin, Opt. Express 15, 14539 (2007).

${ }^{13}$ Z. L. Yuan, A. R. Dixon, J. F. Dynes, A. W. Sharpe, and A. J. Shields, Appl. Phys. Lett. 92, 201104 (2008).

${ }^{14}$ A. R. Dixon, Z. L. Yuan, J. F. Dynes, A. W. Sharpe, and A. J. Shields, Opt Express 16, 18790 (2008).

${ }^{15}$ J. F. Dynes, Z. L. Yuan, A. W. Sharpe, and A. J. Shields, Appl. Phys. Lett. 93, 031109 (2008).

${ }^{16}$ R. Hanbury-Brown and R. Q. Twiss, Nature (London) 177, 4497 (1956).

${ }^{17}$ A. J. Kerman, E. A. Dauler, W. E. Keicher, J. K. W. Yang, K. K. Berggren, G. Gol'tsman, and B. Voronov, Appl. Phys. Lett. 88, 111116 (2006).

${ }^{18}$ B. E. Kardynal, Z. L. Yuan, and A. J. Shields, Nat. Photonics 2, 425 (2008).

${ }^{19}$ S. Moon and D. Y. Kim, Opt. Express 16, 13990 (2008).

${ }^{20} \mathrm{~L}$. Mandel and E. Wolf, Optical Coherence and Quantum Optics (Cambridge University Press, New York, 1995), pp. 712-719. 